\title{
EVALUACIÓN DEL APRENDIZAJE EN LOS PROGRAMAS ORIENTADOS AL REENGANCHE SOCIO-EDUCATIVO E INSERCIÓN LABORAL
}

\author{
Eva MARÍA GonzÁLEZ-BAREA ${ }^{1}$ \\ María Jesús RodrígueZ-Entrena² \\ M. a JosÉ Bolarín-MARTíneZ ${ }^{3}$
}

RESUMEN: En este artículo se analiza la evaluación del alumnado en dos programas de reenganche educativo y social: Programas de Aprendizaje Integral (PAI) y Programas Formativos Profesionales (PFP) en la Región de Murcia (España); se reflexiona sobre el tipo de evaluación y su pertinencia para el reenganche y la inclusión del alumnado. Para ello, se optó por una metodología de investigación de estudios de caso múltiples con diferentes instrumentos de recogida de datos: análisis documental, cuestionario, entrevista y grupos de discusión. Los resultados arrojados en este estudio ratifican el desarrollo de una evaluación en el aula para el aprendizaje a través del uso de distintos instrumentos de evaluación y criterios para evaluar, lo que puede facilitar la inserción educativa y social del alumnado.

Palabras-clave: Reenganche educativo. Evaluación. Aprendizaje. Fracaso escolar. Exclusión socioeducativa.

\section{EVALUATION OF THE LEARNING IN PROGRAMS ORIENTED TOWARDS THE SOCIO- EDUCATIONAL REENGAGEMENT AND LABOR INTEGRATION OF STUDENTS}

\begin{abstract}
In this article the evaluation of students in two educational and social reengagement programs is analyzed: Programas de Aprendizaje Integral (PAI) \& Programas Formativos Profesionales (PFP) in the Region of Murcia (Spain); the type of evaluation and its relevance in the reengagement and inclusion of students is reflected upon. For this, a multiple case study research methodology with different data collection instruments was chosen: documentary analysis, questionnaire, interview and focus groups. The results given in this study ratify the development of an evaluation in the classroom for learning throughout the use of different instruments of evaluation and criterias for evaluating, which can facilitate the educational and social insertion of the student.
\end{abstract}

Keywords: Educational reengagement. Assesment. School failure. Socioeducational exclusion.

Este artículo se enmarca en el proyecto de investigación titulado Procesos de Reenganche Educativo y Sociolaboral de Adolescentes en Situación de Vulnerabilidad. Estudio de Casos e Implicaciones Socioeducativas. EDU2016-76306-C2-1-R. Financiado por el Ministerio de Economía y Competitividad (España, I+D, 2016).

1.Universidad de Murcia - Facultad de Educación - Departamento de Didáctica y Organización Escolar - Murcia, España. E-mail: evamgon@um.es

2.Universidad de Murcia - Facultad de Educación - Departamento de Didáctica y Organización Escolar - Murcia, España. E-mail: entrena@um.es

3.Universidad de Murcia - Facultad de Educación - Departamento de Didáctica y Organización Escolar - Murcia, España. E-mail: mbolarin@um.es

Editor de Sección: Xavier Rambla 


\title{
AVALIAÇÃO DA APRENDIZAGEM EM PROGRAMAS VOLTADOS À REINSCRIÇÃO SOCIOEDUCATIVA E À COLOCAÇÃO DE EMPREGOS
}

\begin{abstract}
RESUMO: Este artigo analisa a avaliação dos alunos em dois programas educacionais e de reinserção social: Programas de Aprendizaje Integral (PAI) e Programas Formativos Profesionales (PFP na Região de Múrcia (Espanha). Reflete-se sobre o tipo de avaliação e sua relevância para o reengajamento e a inclusão dos alunos. Para isso, optou-se por uma metodologia de pesquisa de estudos de casos múltiplos com diferentes instrumentos de coleta de dados: análise documental, questionário, entrevista e grupos de discussão. Os resultados obtidos neste estudo confirmam o desenvolvimento de uma avaliação em sala de aula para a aprendizagem através da utilização de diferentes instrumentos de avaliação e critérios de avaliação, que podem facilitar a inserção educacional e social dos alunos.
\end{abstract}

Palavras-chave: Reengajamento educacional. Avaliação. Aprendizagem. Fracasso escolar. Exclusão socioeducativa.

\section{Introducción}

$\mathrm{E}$ ste artículo se enmarca en el ámbito de los fenómenos de desenganche del alumnado con su formación, abandono educativo y fracaso escolar. Asumimos el planteamiento de Escudero (2005) cuando argumenta que el fracaso escolar es una forma de exclusión educativa que puede implicar y derivar en situaciones de exclusión social (GONZÁLEZ BAREA, 2018) y entendemos a su vez, la existencia de una dinámica dialéctica y multicausal del fracaso escolar (ADAME; SALVÀ, 2010; ESCUDERO, 2005; ESCUDERO; GONZÁLEZ, 2013).

En el momento actual, este tema forma parte de los retos sociales y educativos presentes en las políticas tanto a nivel nacional como internacional. Como parte de la estrategia europea 2020, las políticas educativas tenían como prioridad reducir las cifras del abandono escolar temprano a un máximo del 10\%.

Todavía queda mucho camino por recorrer, pues los datos del 2019 indican que el porcentaje de abandono educativo temprano oscilaba entre los países miembros desde un 3\% en Croacia, hasta un 17,3\% en España ${ }^{1}$. Una cuestión que se agrava en países latinoamericanos, dónde se observa un tímido $18 \%$ en países como Brasil o México (p. e.), poseyendo un alto índice de personas que solo ha alcanzado la educación primaria o la primera etapa de secundaria, un $60 \%$ y un $47 \%$, respectivamente. Un problema que se queda al margen cuando pensamos en los casi dos millones de niños (4 a 17 años) que están sin escolarizar en el país lusófono (NOGUEIRA; PINEDA; PINHEIRO, 2018).

La temática del fracaso escolar y la exclusión sociolaboral de los jóvenes, ha sido puesta en relevancia por organismos internacionales como la OCDE, destacando la importancia de proporcionar a los estudiantes una formación que facilite su desarrollo y su inserción en el mundo laboral. Se trata de una necesidad ampliamente estudiada anteriormente y que se nos muestra en los distintos informes que se vienen facilitando (MINISTERIO, 2019), señalando, en el caso del estado español que solo un 37,3\% de población adulta (2564 años) posee formación postsecundaria terciaria.

Centrándonos en las diferentes Comunidades Autónomas (CCAA) del estado español, cabe indicar que País Vasco $(6,9 \%)$ y Cantabria $(9,8 \%)$ son las que registran las tasas de abandono escolar más bajas de España. En el otro extremo, Andalucía $(24,4 \%)$ y Región de Murcia $(24,1 \%)$ son las que tienen un porcentaje más alto de las 17 CCAA de España. Estas cifran muestran la elevada brecha existente entre Murcia y España, 
presentando una diferencia de 7 puntos (más) respecto a la media del país. En este sentido, es posible destacar algunas peculiaridades de la realidad socioeducativa de esta región que estarían influyendo en la elevada tasa de abandono escolar temprano que presenta:

- Murcia es una de las regiones del país con mayor tasa de paro. La media nacional de parados se encuentra en 16,26\%, siendo en esta comunidad de 17,21\%, según últimos datos publicados por el Instituto Nacional de Estadística $(2020)^{2}$. Esta situación influye en que muchos jóvenes abandonen los estudios para incorporarse al mercado laboral y contribuir así a la economía familiar;

- Esta región se encuentra entre las seis ciudades españolas con la tasa de población extranjera más alta que la media nacional, un 16,14\% frente al 15,90\%, respectivamente. Este escenario se traduce en la presencia de alumnado con desconocimiento de la lengua vehicular del sistema educativo español en las aulas de educación secundaria, lo que supone un gran problema para el aprendizaje y promoción de este alumnado;

- En la etapa de Educación Secundaria, el porcentaje medio de repetidores por curso se sitúa, para todo el territorio nacional, en 9,5\%, siendo Murcia una de las ciudades de España con un porcentaje mucho mayor en repetición, un 14,4\%, según datos del Ministerio de Educación y Formación Profesional (2020). El Sistema Estatal de Indicadores de la Educación (SEIE) resalta que a los 14 años ya ha repetido el 33\% del alumnado matriculado en colegios de Murcia y que a medida que aumenta la edad de los estudiantes va incrementándose la tasa de retraso en los cursos. Gran parte de este alumnado repetidor termina abandonando el sistema educativo ${ }^{3}$.

Ante esta situación, se desarrollan programas, estrategias para mantener en la educación y la formación al alumnado que abandona prematuramente sus estudios. En países como España, Suiza, Portugal y otros, se implementan medidas en entornos escolares y no escolares que evidencian dimensiones y prácticas que tienen éxito y que deberían entenderse como un marco para la reflexión (BOLARÍN, 2020) ante el reto del abandono escolar.

Por ello, la cuestión presentada en este trabajo resulta de un alto interés para países con la problemática que se describe, para la cual, algunos de ellos ya tienen planes propios que ayuden a combatir las distintas dinámicas de exclusión presentes en su sistema educativo. Es el caso de Brasil donde a través del proyecto PROEJA (Programa Nacional de Integración de la Educación Profesional con la Educación Básica en la Modalidad de Educación de Jóvenes y Adultos) ${ }^{4}$, se toman las medidas necesarias para paliar la exclusión que sufren los estudiantes en la enseñanza secundaria, facilitándoles una formación técnica profesional inicial a los jóvenes de 18 a 24 años que contribuya a su desarrollo integral ante la sociedad y el ejercicio efectivo de su ciudadanía (MINISTERIO, 2007). En la creación de este programa, se insistía en la existencia de un grupo numeroso de alumnos con trayectorias escolares que se truncaban ya en la etapa de educación primaria, convirtiéndose en “marginales del sistema educativo" (MINISTERIO, 2007, p. 13), por lo que esta política contra la exclusión se mostró altamente necesaria.

En el marco de este tipo de programas, reparar en la finalidad y en el desarrollo de la evaluación tiene un papel fundamental para que el alumnado más vulnerable y que arrastra trayectorias escolares con dificultades, vuelva a creer en sus posibilidades de aprendizaje y superar desmotivaciones. Asimismo, en función de cómo se conciba la evaluación, ésta puede responder a diferentes finalidades: algunas pedagógicamente ricas (comprender, mejorar, aprender, motivar, diagnosticar...), otras pedagógicamente pobres (medir, comparar, clasificar, acreditar, seleccionar...) y otras con finalidades espurias (jerarquizar, controlar, imponer, castigar...) (MORENO-OLIVOS, 2016; SANTOS GUERRA, 2015). 
Tradicionalmente, la mirada de la evaluación se ha puesto más en los resultados que en los procesos formativos, centrando ésta en aquello que se puede medir. En esta línea, y haciendo referencia a la evaluación de los aprendizajes y a la legislación vigente en España, cabe señalar que la Ley Orgánica para la Mejora de la Calidad Educativa (LOMCE, 2013) incluye los denominados estándares y resultados de aprendizaje evaluables, para medir el grado de adquisición que tiene el alumnado, de las competencias y del logro de los objetivos. Este listado de aprendizajes, que pretende ser observable, evaluable y medible podría tener como consecuencia que todo el desarrollo del currículo derive en una enseñanza por y para la superación de pruebas de evaluación (BOLÍVAR, 2014).

Siguiendo a Santos Guerra (2015), la evaluación supone un proceso de diálogo, compresión y mejora, un diálogo que exige la reflexión conjunta de aquellos que están implicados en la actividad evaluadora. Se aboga por una evaluación no del aprendizaje sino para el aprendizaje (MORENO-OLIVOS, 2016). En esta línea, la evaluación no implica únicamente la consideración de herramientas, momentos, e instrumentos, ésta debe, a su vez, estar alineada y, en coherencia con el resto de elementos del currículum: qué objetivo persigue la enseñanza, cómo hemos desarrollado los contenidos, qué tipo de interacciones hemos favorecido en el aula, etc. Ello implica que el aprendizaje y la evaluación formen parte de un mismo marco conceptual y se entiendan como un continuo (MATEO; VLACHOPOULOS, 2013; BOLARÍN; RODRÍGUEZ-ENTRENA, 2018). Incluso autores como Moreno-Olivos (2016) y Santos Guerra (2015) coinciden en que la evaluación condiciona las decisiones pedagógicas que toman los profesores, así como los aprendizajes del alumnado.

En el caso de los programas de reenganche, Nicol; Thomson; Breslin (2014) hacen hincapié, en base a evidencias de investigaciones sobre buenas prácticas en esta temática (HATTIE, 2009; OCDE, 2005), en la necesidad de utilizar "feedback y formas auténticas de evaluación para construir confianza en la capacidad de aprender" (NICOL; THOMSON; BRESLIN, 2014, p. 21).

En palabras de Trillo, la evaluación auténtica “[...] representa una reacción ante una tradición pedagógica muy extendida en la que el alumno se limita a escuchar, repetir, copiar y memorizar” (2005, p. 91). En esta manera de entender la evaluación se plantean cuestiones problemáticas al alumnado para que este sea capaz de resolverlas. Para favorecer el reenganche real del alumnado que atiende este tipo de programas, se debería prestar una especial atención al riesgo que supone priorizar una enseñanza centrada en lo exclusivamente académico y en las calificaciones escolares que se basen en evaluaciones estandarizadas y descontextualizadas de los estudiantes (INGLÉS et al., 2011; BOLARÍN; RODRÍGUEZ-ENTRENA, 2018).

En esta línea y, en coherencia con las propuestas de aula, se deberían valorar no solo conocimientos (conceptos), sino las aplicaciones del mismo (procedimientos) y actitudes, lo que lleva al docente a integrar estrategias de evaluación y estrategias de aprendizaje (MARCELO; RIVERA, 2017). Para ello, se hace necesario plantear evaluaciones mediante actividades, tareas y/o problemas próximos a la realidad del alumnado y a los contenidos trabajados (casos prácticos), donde la curiosidad y la utilidad de lo aprendido tienen una finalidad formativa en la sesión de enseñanza-aprendizaje a la vez que permiten los reajustes en las mismas (FRADE, 2008).

Por otra parte, cabe resaltar la importancia del uso de una variedad de instrumentos que garanticen el desarrollo de una evaluación formativa y auténtica. Si bien, a lo largo del proceso de enseñanza y aprendizaje, un mismo instrumento puede ser utilizado en función de diferentes pretensiones y finalidades: realizar diagnósticos, regular aprendizajes, acreditar y cualificar, o bien, evaluar saberes, procesos, competencias, comportamientos y actitudes, etc. (SANMARTÍ, 2020; CASTEJÓN et al., 2019). Entre los muchos instrumentos posibles, destacamos, por una parte, las pruebas objetivas y semiobjetivas y/o de desarrollo escrito, que resultan útiles para evaluar contenidos diversos, amplios o relacionados entre sí a nivel conceptual. Igualmente, aquellas pruebas que requieran la solución de problemas y/o casos procedimentales son apropiados para la evaluación de la comprensión y el nivel de razonamiento del alumnado. Por último, resaltamos el registro de la observación conductual que puede ser muy recomendable para el desarrollo de una evaluación continua de actitudes (MONTANERO, 2019). 
En base al marco teórico planteado, en este artículo se analizan las prácticas de evaluación desarrolladas que están contribuyendo a fortalecer los aprendizajes del alumnado y por extensión, contribuyendo al reenganche y la inclusión socio-laboral del alumnado. Concretamente, se reflexiona sobre la conceptualización y el desarrollo de la evaluación de los aprendizajes de los estudiantes, dentro de dos programas destinados al reenganche socioeducativo en España: Programa de Aprendizaje Integral (en adelante, PAI), y Programa Formativo Profesional en modalidad adaptada (en adelante, PFP). Estos programas tienen como destinatarios al alumnado que bien, ha abandonado la Educación Secundaria Obligatoria (ESO) sin titular, o bien la está cursando y no está en condiciones de promocionar, o muestra un rechazo escolar, absentismo o incorporación tardía al sistema educativo, desfase curricular generalizado, etc., debido a historias personales o a trayectorias escolares previas.

\section{Método}

\section{Diseño Metodológico}

El diseño metodológico de la investigación se centró en un estudio de casos múltiples, que integra procedimientos cuantitativos y cualitativos, que consiste en una estrategia de investigación que "estudia varios casos únicos al mismo tiempo para comprender la realidad que desea explorar, describir, explicar, evaluar o modificar" (LÓPEZ GONZÁLEZ, 2013, p. 141). Es decir, para “analizar aquellos problemas o situaciones que presentan múltiples variables y que están estrechamente vinculados al contexto en el que se desarrollan” (CEBREIRO; FERNÁNDEZ MORANTE, 2004, p. 667). Por tanto, más allá de la cantidad se ha priorizado la calidad: que cada unidad seleccionada que conforme la muestra total facilite el acceso a datos profundos, en detalle y realmente ricos para nuestro foco de interés (MARTÍNEZ-SALGADO, 2012; VILLEGAS; GONZÁLEZ, 2011).

La unidad de análisis fueron los programas formativos que han tenido éxito en el reenganche socioeducativo del alumnado que lo cursa y, por tanto, contribuyen a aminorar los riesgos del abandono educativo y formativo. En la siguiente Tabla 1 se presenta una información descriptiva sobre los programas objeto de análisis.

Respecto al proceso de selección de los casos, este siguió diferentes fases:

- En una primera fase se realizó un análisis sobre las propuestas formativas (programas) -desarrolladas en centros escolares, instituciones municipales, ONGs y otras instancias de formación- en el ámbito de la Comunidad Autónoma de la Región de Murcia en materia de reenganche socio-educativo (mayo a julio de 2017).

- En una segunda fase, se seleccionaron las ofertas formativas (programas) que obtienen mejores resultados (septiembre a diciembre de 2017).

- Posteriormentey ya en una tercera fase, se procedió a seleccionar los centros. Esta selección se realizó en base a los siguientes criterios: nivel de efectividad del programa (en términos de reenganche educativo y/o inserción laboral), la pluralidad de marcos organizativos, diversidad de la población joven a la que se dirigen, la estabilidad de los programas y la receptividad de las instituciones.

- A continuación y en una cuarta fase, se contactó con las instituciones responsables para la recogida de información y se procedió a la recogida de la misma (marzo a julio de 2018).

En concreto, las unidades de estudio de caso que incluimos en este artículo se refieren al PAI desarrollado en un centro de educación secundaria y el PFP desarrollado en instituciones sociales de la Región de Murcia. Del PFP se analizaron tres perfiles formativos desarrollados por dos ONGs (Fundación X y Asociación Y). 
Tabla 1. Descripción de los programas

\begin{tabular}{ccc}
\hline Programas/ & PAI & PFP \\
Características & PA \\
\hline
\end{tabular}

Estudiante de $2^{\circ}$ de ESO que: valore negativamente el marco escolar y con serias dificultades de adaptación al medio y no reúna los requisitos de acceso a enseñanzas de Formación Profesional. Con 14 años o cumplidos en el año en que accede.

$\begin{array}{cl}\begin{array}{c}\text { Entidades de } \\ \text { oferta }\end{array} & \begin{array}{l}\text { Centros educativos públicos y concertados } \\ \text { que impartan ESO y que lo soliciten. }\end{array}\end{array}$

Finalidad

Favorecer su integración en el centro educativo y promover el desarrollo de las competencias básicas de la etapa. Reducir el absentismo y disminuir el riesgo de abandono.

Estructura Organización en ámbitos y materias curricular (ver Tabla 4)

Continua, formativa e integradora

Evaluación (ver Resultados: Evaluación del alumnado desde la normativa)
Estudiantes que cursen y no estén en condiciones de promocionar, o muestren rechazo al ámbito escolar, que evidencie riesgo de exclusión del sistema. Con 16 y 21 años, cumplidos en el año natural de incorporación.

Entidades sin ánimo de lucro legalmente constituidas, con experiencia acreditada en atención a jóvenes socialmente desfavorecidos. Adscritos a IES (Institutos de Educación Secundaria) a efectos de certificación y custodia de expedientes académicos.

Dotar al alumnado de las competencias personales, sociales y profesionales adecuadas a sus características y necesidades que favorezcan su inserción sociolaboral y su incorporación a la vida activa con responsabilidad y autonomía.

Organización en módulos profesionales de ciclo formativo de formación profesional básica (ver Tabla 5)

Continua, formativa e integradora y se realizará teniendo como referencia los objetivos, expresados como resultados de aprendizaje, así como las competencias profesionales, personales y sociales y el grado de madurez alcanzado en los respectivos módulos en relación con los objetivos indicados en el artículo 4 de la normativa reguladora del programa.

Duración

Un curso académico

Tamaño del grupo

Mínimo 10, máximo 15 alumnos.

Certificado académico del Programa Formativo Profesional o -si no se supera- Certificado de las unidades de competencia adquiridas, así como los

Titulación Ninguna específica módulos de Ciencias Aplicadas y Comunicación y Sociedad superados. Posibilidades de solicitar certificado profesional.

a) Grupo ordinario del curso que corresponda.

Continuación formación b) $2^{\circ}$ curso del Programa de Mejora del Aprendizaje y Rendimiento.

c) Formación Profesional; entre otras. a) Nivel II de Educación Secundaria para

Personas Adultas.

b) Módulos que le falten para obtener un título. de Formación Profesional Básica. 


\section{Fuentes de Información}

Las fuentes de información incluyen la evidencia documental, alumnado, profesorado y otros agentes educativos implicados en los citados programas (pedagogos y equipo directivo). Esta información se recoge en las Tablas 2 y 3 :

Tabla 2. Fuentes de información del PAI y PFP. Alumnado

\begin{tabular}{ccccc}
\hline Entidades & $\begin{array}{c}\text { PAI/ PFP. } \\
\text { Perfiles Formativos }\end{array}$ & $\begin{array}{c}\text { Total } \\
\text { matriculados }\end{array}$ & $\begin{array}{c}\text { Participantes. } \\
\text { Proceso cuantitativo }\end{array}$ & $\begin{array}{c}\text { Participantes. } \\
\text { Proceso cualitativo }\end{array}$ \\
\hline IES A & 12 & $12(100 \%)$ & $12(100 \%)$ \\
\hline \multirow{2}{*}{ Fundación X } & $\begin{array}{c}\text { Operaciones Auxiliares de } \\
\text { Revestimiento en Construcción }\end{array}$ & 12 & $8(66,6 \%)$ & $4(33,3 \%)$ \\
\hline \multirow{2}{*}{ Asociación Y } & Servicios Auxiliares de Peluquería & 15 & $7(46,6 \%)$ & $11(73,3 \%)$ \\
\cline { 2 - 5 } & Operaciones Básicas Ofimática & 15 & $5(33,3 \%)$ & 0 \\
\hline & Total & 42 & 20 & 15 \\
\hline
\end{tabular}

Fuente: Elaboración propia.

Tabla 3. Fuentes de Información PAI y PFP. Profesorado y otros agentes educativos

\begin{tabular}{cccccc}
\hline Instituciones & PAI y PFP. Perfiles Formativos & $\begin{array}{c}\text { Profesorado } \\
\text { participante }\end{array}$ & $\begin{array}{c}\text { Profesorado } \\
\text { total }\end{array}$ & Otros agentes \\
\hline IES A & - & 2 & 4 & PTSC y Director del Centro \\
\hline \multirow{2}{*}{ Fundación X } & $\begin{array}{c}\text { Operaciones Auxiliares de } \\
\text { Revestimiento en Construcción }\end{array}$ & 2 & 3 & Pedagoga \\
\hline \multirow{2}{*}{ Asociación $\mathrm{Y}^{5}$} & Servicios Auxiliares de Peluquería & 3 & 3 & \multirow{2}{*}{ Directora } \\
\cline { 2 - 5 } & Operaciones Básicas Ofimática & 3 & 3 & \\
\hline
\end{tabular}

Fuente: Elaboración propia.

\section{Instrumentos y Procedimientos de Recogida de Información}

Los instrumentos de recogida de datos fueron:

- Registro de documentos que aportan información sobre los programas y la normativa que regula.

- Entrevistas en profundidad a los participantes en los programas objeto de estudio. El guión de entrevista incluía diferentes apartados:

1. Cuestiones generales sobre el programa y el propio informante;

2. Alumnado;

3. Documentos formales del centro/planes/programaciones...;

4. Coordinación;

5. El trabajo con el alumnado en el aula;

6. El profesor y sus circunstancias laborales. 
- Cuestionario para el alumnado. El cuestionario se dividió en cinco dimensiones:

1. Datos de identificación;

2. Trayectoria escolar e incorporación al programa;

3. El programa y qué se hace en él;

4. Las clases y las relaciones. En las que se incluían 20 preguntas cerradas, tipo Likert de cinco opciones de respuesta con grado de frecuencia $(1=$ nunca; $5=$ siempre $)$ y de acuerdo ( 1 = nada de acuerdo; 5 = muy de acuerdo).

- Grupos de discusión con el alumnado implicado. Los interrogantes y temáticas que se incluyeron se dividieron en:

1. Experiencia escolar previa del alumnado que está cursando el programa;

2. Proceso de desenganche e incorporación al programa;

3. Experiencia en el programa;

4. Incidencia del programa en el alumnado y

5. Perspectivas de futuro.

Todos estos instrumentos fueron validados por expertos -docentes universitarios- con amplia trayectoria investigadora sobre la temática. Las aportaciones y sugerencias de los expertos se incluyeron en los instrumentos definitivos con el fin de conseguir la validez y fiabilidad de los mismos.

Los datos se recogieron durante los cursos 2016-2017 y 2017-2018, una vez realizados los contactos previos necesarios. Las entrevistas y grupos de discusión se grabaron y transcribieron para su posterior análisis, respetando el protocolo de confidencialidad y contando con el informe favorable del Comité de Ética de la Universidad de Murcia. Los cuestionarios fueron realizados por los estudiantes con el/la tutor/a en sus centros respectivos.

\section{Análisis de Datos}

Los datos de carácter cuantitativo y cualitativo fueron analizados, respectivamente, a través de estadística descriptiva (frecuencias y porcentajes) aplicando el paquete estadístico Statistical Package for the Social Sciencies (SPSS, versión 24) y la técnica de análisis de contenido cualitativo (MILES; HUBERMAN; SALDAÑA, 2014) mediante la delimitación de unidades de análisis, codificación abierta y categorización, apoyándonos en el programa informático ATLAS.ti, versión 8.

\section{Resultados}

\section{La Evaluación del Alumnado desde la Normativa}

En el caso del PAI, la evaluación del alumnado siguiendo la normativa será continua, formativa e integradora y contempla como elementos curriculares los criterios de evaluación, entendidos como el referente específico para evaluar el aprendizaje del alumnado. Los conocimientos y competencias recogidos en la normativa reflejan aquello que se debe alcanzar por el alumnado y que será evaluable. Estos deberán ser observables, medibles y evaluables, así como permitir graduar el rendimiento o logro alcanzado. Por otro lado, su diseño debe contribuir y facilitar el diseño de pruebas estandarizadas y comparables. Además, se priorizarán los aprendizajes que tengan un mayor carácter aplicado a situaciones de la vida cotidiana. Puesto 
que el currículum en el PAI está organizado por ámbitos, el alumnado tendrá una calificación única para cada uno de los ámbitos y materias y se atendrá a los criterios establecidos en la programación de los mismos (BOLARIN; RODRÍGUEZ-ENTRENA, 2018). En la Tabla 4 se incluye la organización curricular de este programa.

Tabla 4. Organización de materias en ámbitos de conocimiento ${ }^{6}$

\begin{tabular}{cc}
\hline Ámbito & Materias que incluye \\
\hline Ciencias aplicadas & Biología y Geología, Física y Química y Matemáticas \\
\hline Sociolingüístico & Geografía e Historia y Lengua castellana y Literatura \\
\hline Artístico & Educación plástica, visual y audiovisual y Música \\
\hline Motriz y de expresión corporal & Educación física y contenidos de Expresión Corporal \\
\hline Primera Lengua Extranjera & \\
\hline Religión o Valores éticos & \\
\hline
\end{tabular}

Fuente: Elaboración propia.

El equipo docente podrá determinar, en circunstancias excepcionales, la promoción de un alumno con evaluación negativa en tres materias o ámbitos, también podrá proponer que el alumno que no esté en condiciones de promocionar pueda cursar por segunda vez este programa.

En lo referente al PFP, el currículo queda dividido en módulos, siendo la evaluación diferente para cada uno de ellos (Tabla 5).

Tabla 5. Organización modular de los PFP

\begin{tabular}{cc} 
Módulos asociados a competencias & Unidades de competencia de Cualificaciones Profesionales Nivel $\mathbf{1}$ \\
\hline \multirow{3}{*}{ Módulos no asociados competencias } & Comunicación y Sociedad \\
\cline { 2 - 2 } & Ciencias Aplicadas \\
\hline
\end{tabular}

Módulo de Formación en Centros de Trabajo

Fuente: Elaboración propia.

En el caso de los Módulos asociados a unidades de competencia, para la evaluación del aprendizaje del alumnado se tendrá en cuenta lo establecido en los diferentes títulos y currículos de Formación Profesional. Igualmente, para los Módulos no asociados a unidades de competencia, la evaluación del alumnado partirá de los criterios de evaluación y resultados de aprendizaje detallados en los módulos de Comunicación y Sociedad I y Ciencias Aplicadas I de los títulos y currículos de Formación Profesional. En estos módulos y para el caso de los módulos optativos, la evaluación se regirá por diferentes criterios respondiendo a las distintas materias que se trate. En lo referente a la evaluación del Módulo de Formación en Centros de Trabajo se indica que, para poder cursar este módulo, el alumnado obligatoriamente debe haber superado todos los módulos asociados a unidades de competencia, no ocurre lo mismo para el caso de los módulos no asociados a competencias. Estos últimos no necesariamente deben contar con evaluación positiva previa a la realización del Módulo de Formación en Centros de Trabajo. Por otra parte, tendrá una convocatoria extraordinaria para la superación de ambos módulos: aquellos no asociados a competencia y aquellos otros asociados a unidades de competencia. Por último, cabe indicar que, en el Módulo de Formación en Centros 
de Trabajo, la evaluación se realizará al final del último trimestre del curso del programa. No obstante, su realización quedará exenta para el alumnado que pueda acreditar experiencia laboral de, al menos, 6 meses en una actividad que guarde relación con el PFP que cursa.

Como información general para la evaluación del PFP se indica en la normativa al respecto que se llevará a cabo una evaluación inicial para detectar el nivel en torno a las actitudes, competencias, capacidades y conocimientos básicos del alumnado. Teniendo en cuenta el resultado de esta evaluación, el profesorado desarrollará tres sesiones de evaluación a lo largo del curso -coordinadas por el/la tutor/a-, la última de ellas previa a la incorporación del alumnado al Módulo de Formación en Centros de Trabajo.

Por último, la normativa permite la repetición - una sola vez- del PFP por parte del alumnado que no lo haya superado.

\section{La Evaluación en las Aulas}

\section{La Evaluación en el PAI}

Se presentan los datos recogidos -a través de la realización de cuestionarios- sobre las tareas que lleva a cabo el alumnado siguiendo sus propios argumentos (Tabla 6).

Tabla 6. Tareas realizadas por el alumnado. PAI

\begin{tabular}{|c|c|c|c|c|c|}
\hline Tareas & $\begin{array}{c}1 \\
\text { Nunca }\end{array}$ & $\begin{array}{c}2 \\
\text { Casi nunca }\end{array}$ & $\begin{array}{c}3 \\
\text { Algunas veces }\end{array}$ & $\begin{array}{c}4 \\
\text { Bastantes veces }\end{array}$ & $\begin{array}{c}5 \\
\text { Casi siempre }\end{array}$ \\
\hline Comprender bien los temas y contenidos & $8,3 \%$ & & $16,6 \%$ & $58,3 \%$ & $16,6 \%$ \\
\hline Memorizar los contenidos & $16,6 \%$ & $33,3 \%$ & $33,3 \%$ & $8,3 \%$ & $8,3 \%$ \\
\hline Hacer ejercicios y trabajos solo/a & $16,6 \%$ & $16,6 \%$ & $8,3 \%$ & $16,6 \%$ & $33,3 \%$ \\
\hline Hacer, en grupo trabajos, ejercicios & $25 \%$ & $33,3 \%$ & $8,3 \%$ & $25 \%$ & $8,3 \%$ \\
\hline Utilizar o aplicar los aprendizajes & $16,6 \%$ & $25 \%$ & $16,6 \%$ & $41 \%$ & \\
\hline Razonar, pensar y resolver problemas & $8,3 \%$ & $8,3 \%$ & $25 \%$ & $41 \%$ & $16,6 \%$ \\
\hline Corregir ejercicios con el docente & $8,3 \%$ & & $41 \%$ & $16,6 \%$ & $33,3 \%$ \\
\hline
\end{tabular}

Fuente: Elaboración propia.

Como se puede observar en la Tabla 6, en primer lugar, las respuestas acerca de la comprensión de los temas y los contenidos que se trabajan alcanza un mayor porcentaje -un $80 \%$. Este resultado queda avalado también por el alto porcentaje registrado en la frecuencia de nunca y casi nunca en la tarea enunciada como memorización de los contenidos. Esto es, el alumnado indica que casi nunca memoriza el contenido, si bien, le otorga gran importancia a su comprensión.

Respecto a la realización de tareas y actividades, el alumnado indica en un $50 \%$ que bastantes veces y casi siempre se llevan a cabo individualmente, indicando, al mismo tiempo, que en un 58\% nunca y casi nunca se realizan de forma grupal. Si bien, existe un casi $9 \%$ de respuestas que indican que sólo algunas veces se realizan tareas en ambas modalidades -individual y grupal.

Referente a las tareas de razonar, pensar y resolver problemas, los datos evidencian que el alumnado lo lleva a cabo, bastantes veces y casi siempre, en un 57.6\%. En este sentido, cabe tener en cuenta que un $25 \%$ responden que ello sólo sucede a veces.

En cuanto a la aplicación y/o utilización de los aprendizajes adquiridos por parte del alumnado, este 
indica un porcentaje similar entre las respuestas distribuidas en las frecuencias nunca y casi nunca -41,6\%y casi siempre $-41 \%$. En este sentido, sólo un 16,6\% responde que a veces existe un uso de los aprendizajes.

Por último, un $50 \%$ del alumnado responde que bastantes veces y casi siempre corrige los ejercicios con los/as docentes. Sin embargo, un $41 \%$ de las respuestas se posicionan en la frecuencia de algunas veces.

Siguiendo con el PAI, a continuación, se presentan los datos recogidos sobre las tareas a las que presta una especial atención el profesorado (Tabla 7), teniendo en cuenta las respuestas del alumnado sobre ello. En general, los resultados muestran porcentajes altos en las respuestas positivas en torno a la mayoría de los ítems. De esta forma, y para las frecuencias de bastante y mucha importancia, la mayoría de respuestas del alumnado se encuentran en las tareas de atender a las explicaciones en clase, memorizar los contenidos explicados en el aula y conocer su evaluación y aquellos aspectos a mejorar, todos ellos comparten un total de $83,3 \%$ de las respuestas en las frecuencias señaladas. Con un porcentaje muy similar, un $82 \%$, el alumnado indica que con bastante y mucha importancia los/as docentes valoran la realización de actividades y ejercicios prácticos, así como la comprensión correcta de los temas trabajados.

Con un porcentaje algo menor, un 74,9\%, el profesorado presta atención a la participación del alumnado en clase. Menos respuestas se concentran en el ítem referente a la preparación de los exámenes y pruebas, donde existen el 66,6\% de las respuestas para las frecuencias de bastante y mucha importancia. Si bien, la tarea menos atendida por el profesorado según las respuestas del alumnado, consiste en la exposición de trabajos en clase, dado que el 50\% de los/as estudiantes indicaron que su importancia era ninguna o poca.

En cuanto a los valores y conductas que el profesorado valora, las respuestas del alumnado son elevadas en las frecuencias de bastante y mucha importancia, manifestando que se valoran las buenas relaciones con compañeros/as así como tener buena conducta en clase.

Tabla 7. Atención prestada por el profesorado. PAI

\begin{tabular}{|c|c|c|c|c|c|}
\hline & $\begin{array}{c}1 \\
\text { Ninguna } \\
\text { importancia }\end{array}$ & $\begin{array}{c}2 \\
\text { Poca } \\
\text { importancia }\end{array}$ & $\begin{array}{c}3 \\
\text { Alguna } \\
\text { importancia }\end{array}$ & $\begin{array}{c}4 \\
\text { Bastante } \\
\text { importancia }\end{array}$ & $\begin{array}{c}5 \\
\text { Mucha } \\
\text { importancia }\end{array}$ \\
\hline Prestar atención a las explicaciones & & & & $25 \%$ & $58,3 \%$ \\
\hline Memorizar los contenidos explicados en clase & & $16,6 \%$ & & $58,3 \%$ & $25 \%$ \\
\hline Comprender bien los temas & & & & $41 \%$ & $41 \%$ \\
\hline Realizar actividades prácticas o ejercicios & & & & $41 \%$ & $41 \%$ \\
\hline Exponer trabajos en clase & $25 \%$ & $25 \%$ & & $8,3 \%$ & $33,3 \%$ \\
\hline Participar en clase cuando se trabaje algún tema & & $8,3 \%$ & & $16,6 \%$ & $58,3 \%$ \\
\hline Tener buena relación con compañeros/as & & & & $33,3 \%$ & $58,3 \%$ \\
\hline Conocer mi evaluación en las clases y qué mejorar & & & & $33,3 \%$ & $50 \%$ \\
\hline Preparar bien los exámenes o pruebas & $8,3 \%$ & & & $33,3 \%$ & $33,3 \%$ \\
\hline Tener buena conducta en clase & & & & $25 \%$ & $50 \%$ \\
\hline
\end{tabular}

Fuente: Elaboración propia

Por otra parte, los datos cualitativos recabados en esta investigación apuntan a que la devolución de la evaluación al alumnado es una cuestión de suma importancia en la enseñanza en general y, en el desarrollo de este tipo de programas en particular. Se trata de una evaluación enfocada al aprendizaje del alumnado, una evaluación formativa que permite a los/as estudiantes conocer sus progresos académicos, así como también ser conscientes de los fallos y cuestiones a mejorar en su propio aprendizaje. 
A la luz de los resultados, cabe indicar que la práctica evaluativa no se centra únicamente en competencias y conocimientos observables, medibles y evaluables que permiten graduar el rendimiento o logro alcanzado. El profesorado implicado valora actitudes y comportamientos que se entienden como indicadores de reenganche y, que permiten hablar de una ausencia progresiva de rechazo al entorno escolar, causa del fracaso del alumnado que participa en este programa. Así, se valora lo "actitudinal" y lo "curricular". El alumnado indica, tal y como se ha señalado anteriormente, corregir los ejercicios con sus profesores/as, asimismo, señalan la gran importancia que sus docentes otorgan a que ellos conozcan su evaluación en las clases, así como aquellos aspectos a solventar, para la mejora de sus aprendizajes. En esta línea, el tutor del PAI pone de manifiesto el valor formativo que tienen para el alumnado los distintos instrumentos de evaluación y la devolución de información procedente de los mismos:

El alumnado participa, pregunta, se involucra en la corrección del examen o en la actividad o cómo voy, al fin y al cabo, tu trabajo es que ellos realicen la tarea mejor o peor, pero que la empiecen y la terminen [...] una prueba escrita dentro de un plan de planificación e integración ha de servir para que ellos aprendan, es decir, es un proceso de aprendizaje (Tutor, PAI).

Igualmente, los datos recogidos -en el PAI- ponen de manifiesto la diversidad de instrumentos utilizados por el profesorado para la evaluación de los aprendizajes del alumnado. En este sentido, el tutor y la profesora de inglés del PAI indican:

[...] pruebas escritas, pruebas orales, exposiciones, trabajos, proyectos, trabajos en el huerto o un proyecto en el taller construcción de algún objeto (Tutor, PAI).

Observación directa, pruebas escritas y orales. En las escritas entran diferentes destrezas (Profesora Inglés, PAI).

Respecto al qué se evalúa, los/as docentes expresan la multitud de aspectos que son objeto de la evaluación, atendiendo no sólo a cuestiones estrictamente académicas y/o curriculares sino también a aquellas otras referentes a las habilidades personales y actitudes.

Se valora la realización de las actividades, la predisposición a hacerlas, la perseverancia, no es más el resultado como el camino, cómo ha llegado, cómo ha evolucionado el alumno ante la actividad, ante todo lo que se ha hecho (Tutor, PAI).

Tenemos en cuenta la participación. También se evalúa el aula, el trabajo en clase y casa y tomo nota todos los días (Profesora de Inglés, PAI).

\section{La Evaluación en el PFP}

En el caso del PFP y, concretamente, en los Módulos asociados a unidades de competencia, los resultados -teniendo en cuenta las respuestas de los/as estudiantes- ponen de manifiesto que el alumnado valora en las frecuencias de bastantes veces y casi siempre la mayoría de las afirmaciones que se incluían en el cuestionario. De esta forma, en un $90 \%$, el mayor porcentaje recogido, los/as alumnos/as indican que 
hacen ejercicios y trabajos de forma individual, así como que aplican los aprendizajes adquiridos en bastantes veces y casi siempre. En un porcentaje ligeramente inferior, el $85 \%$ del alumnado afirma que bastantes veces y casi siempre memorizan los contenidos, razonan, piensan y resuelven problemas, así como que llevan a cabo la corrección de ejercicios con los/as docentes. Respecto a la realización de ejercicios y trabajos en grupo, el alumnado indica, en un $80 \%$, que lo llevan a cabo en bastantes veces y casi siempre. En último lugar, el porcentaje más bajo entre todos los ítems referentes a las tareas que realiza el alumnado, el $75 \%$, se concentra en la afirmación comprender bien los temas y contenidos en la frecuencia de bastantes veces y casi siempre.

Cabe indicar que, en el caso de los módulos no asociados a unidades de competencia, las valoraciones del alumnado en los ítems del cuestionario, muestran porcentajes muy similares a los presentados, no encontrándose diferencias significativas, tal y como puede comprobarse en la Tabla 8.

Tabla 8. Tareas realizadas por el alumnado. PFP

\begin{tabular}{|c|c|c|c|c|c|}
\hline \multirow[b]{2}{*}{ Tareas } & \multicolumn{5}{|c|}{ Módulos asociados a competencia } \\
\hline & $\begin{array}{c}1 \\
\text { Nunca }\end{array}$ & $\begin{array}{c}2 \\
\text { Casi nunca }\end{array}$ & $\begin{array}{c}3 \\
\text { Algunas veces }\end{array}$ & $\begin{array}{c}4 \\
\text { Bastantes veces }\end{array}$ & $\begin{array}{c}5 \\
\text { Casi siempre }\end{array}$ \\
\hline Comprender bien los temas y contenidos & & & $20 \%$ & $25 \%$ & $50 \%$ \\
\hline Memorizar los contenidos & & & $10 \%$ & $40 \%$ & $45 \%$ \\
\hline Hacer ejercicios y trabajos solo/a & 0 & $5 \%$ & $5 \%$ & $35 \%$ & $55 \%$ \\
\hline Hacer, en grupo trabajos, ejercicios & 0 & $10 \%$ & $10 \%$ & $15 \%$ & $65 \%$ \\
\hline Utilizar o aplicar los aprendizajes & & & $10 \%$ & $35 \%$ & $55 \%$ \\
\hline Razonar, pensar y resolver problemas & & & $15 \%$ & $35 \%$ & $50 \%$ \\
\hline Corregir ejercicios con el docente & $5 \%$ & $5 \%$ & $5 \%$ & $35 \%$ & $50 \%$ \\
\hline \multirow[b]{2}{*}{ Tareas } & \multicolumn{5}{|c|}{ Módulos no asociados a competencia } \\
\hline & $\begin{array}{c}1 \\
\text { Nunca }\end{array}$ & $\begin{array}{c}2 \\
\text { Casi nunca }\end{array}$ & $\begin{array}{c}3 \\
\text { Algunas veces }\end{array}$ & $\begin{array}{c}4 \\
\text { Bastantes veces }\end{array}$ & $\begin{array}{c}5 \\
\text { Casi siempre }\end{array}$ \\
\hline Comprender bien los temas y contenidos & & & $20 \%$ & $25 \%$ & $45 \%$ \\
\hline Memorizar los contenidos & $5 \%$ & & $15 \%$ & $35 \%$ & $40 \%$ \\
\hline \multicolumn{6}{|l|}{ Hacer ejercicios y trabajos solo/a } \\
\hline \multicolumn{6}{|l|}{ Hacer, en grupo trabajos, ejercicios } \\
\hline Utilizar o aplicar los aprendizajes & & $10 \%$ & $5 \%$ & $10 \%$ & $70 \%$ \\
\hline Razonar, pensar y resolver problemas & & $5 \%$ & $20 \%$ & $15 \%$ & $55 \%$ \\
\hline Corregir ejercicios con el docente & & & & & \\
\hline
\end{tabular}

Fuente: Elaboración propia.

Tal y como se recoge en la Tabla 9, la totalidad de las respuestas del alumnado acerca de los aspectos a los que el profesorado presta atención en los módulos asociados a unidades de competencia, se concentran en las frecuencias de bastante y mucha importancia, con unos porcentajes que oscilan entre el 75 y el 100\%. En dicha horquilla se sitúa el ítem participar en clase cuando se trabaje algún tema, para el porcentaje menor, y tener buena conducta en clase para el porcentaje mayor. Entre esos porcentajes, destacamos prestar atención a las explicaciones, realizar actividades prácticas o ejercicios, conocer la evaluación y aspectos a mejorar, preparar bien los exámenes y pruebas, con un 95\% de respuestas para las frecuencias mencionadas. En otra dirección, los resultados evidencian menores porcentajes -aunque respetando la horquilla de referencia- en las tareas de participación en clase y comprender bien los temas. 
Se puede resaltar que, en el caso de los módulos no asociados a unidades de competencia, las valoraciones del alumnado respecto a las cuestiones que el profesorado valora, muestran porcentajes muy similares a los presentados, no encontrándose tampoco diferencias significativas. Sin embargo, resalta el mayor porcentaje de respuestas, para las frecuencias de bastante y mucha importancia, en el ítem comprender bien los temas en estos módulos frente a los módulos asociados a unidades de competencia -un 95\% frente al 80\%. Si bien también es cierto la ausencia de un $10 \%$ de respuestas para este ítem en el caso de los módulos asociados a unidades de competencia.

Tabla 9. Atención prestada por el profesorado. PFP

\begin{tabular}{|c|c|c|c|c|c|}
\hline & \multicolumn{5}{|c|}{ Módulos asociados a competencia } \\
\hline & $\begin{array}{c}1 \\
\text { Ninguna } \\
\text { importancia }\end{array}$ & $\begin{array}{c}2 \\
\text { Poca } \\
\text { importancia }\end{array}$ & $\begin{array}{c}3 \\
\text { Alguna } \\
\text { importancia }\end{array}$ & $\begin{array}{c}4 \\
\text { Bastante } \\
\text { importancia }\end{array}$ & $\begin{array}{c}5 \\
\text { Mucha } \\
\text { importancia }\end{array}$ \\
\hline Prestar atención a las explicaciones & & & $5 \%$ & $55 \%$ & $40 \%$ \\
\hline Memorizar los contenidos explicados en clase & & & $5 \%$ & $45 \%$ & $40 \%$ \\
\hline Comprender bien los temas & & $10 \%$ & & $55 \%$ & $35 \%$ \\
\hline Realizar actividades prácticas o ejercicios & & & $5 \%$ & $50 \%$ & $45 \%$ \\
\hline Exponer trabajos en clase & $5 \%$ & & $15 \%$ & $50 \%$ & $40 \%$ \\
\hline Participar en clase cuando se trabaje algún tema & & $5 \%$ & $10 \%$ & $20 \%$ & $65 \%$ \\
\hline Tener buena relación con compañeros/as & & $10 \%$ & $5 \%$ & $35 \%$ & $45 \%$ \\
\hline Conocer mi evaluación en las clases y qué mejorar & & & $5 \%$ & $50 \%$ & $45 \%$ \\
\hline Preparar bien los exámenes o pruebas & & & & $60 \%$ & $35 \%$ \\
\hline \multirow[t]{3}{*}{ Tener buena conducta en clase } & & & & $30 \%$ & $70 \%$ \\
\hline & \multicolumn{5}{|c|}{ Módulos no asociados a competencia } \\
\hline & $\begin{array}{c}1 \\
\text { Ninguna } \\
\text { importancia }\end{array}$ & $\begin{array}{c}2 \\
\text { Poca } \\
\text { importancia }\end{array}$ & $\begin{array}{c}3 \\
\text { Alguna } \\
\text { importancia }\end{array}$ & $\begin{array}{c}4 \\
\text { Bastante } \\
\text { importancia }\end{array}$ & $\begin{array}{c}5 \\
\text { Mucha } \\
\text { importancia }\end{array}$ \\
\hline Prestar atención a las explicaciones & & & $15 \%$ & $35 \%$ & $50 \%$ \\
\hline Memorizar los contenidos explicados en clase & & & $10 \%$ & $45 \%$ & $45 \%$ \\
\hline Comprender bien los temas & & $5 \%$ & & $55 \%$ & $40 \%$ \\
\hline Realizar actividades prácticas o ejercicios & & & $5 \%$ & $50 \%$ & $45 \%$ \\
\hline Exponer trabajos en clase & & & & $50 \%$ & $30 \%$ \\
\hline Participar en clase cuando se trabaje algún tema & $5 \%$ & $5 \%$ & $5 \%$ & $10 \%$ & $75 \%$ \\
\hline Tener buena relación con compañeros/as & & $10 \%$ & $5 \%$ & $25 \%$ & $60 \%$ \\
\hline Conocer mi evaluación en las clases y qué mejorar & & & $10 \%$ & $35 \%$ & $55 \%$ \\
\hline Preparar bien los exámenes o pruebas & & & $15 \%$ & $35 \%$ & $40 \%$ \\
\hline Tener buena conducta en clase & & & & $30 \%$ & $70 \%$ \\
\hline
\end{tabular}

Fuente: Elaboración propia.

La información recabada pone de manifiesto una práctica evaluativa caracterizada por una evaluación continua, con diferentes aspectos a evaluar, tanto académicos como conductuales, el tener en cuenta distintos porcentajes en los diversos instrumentos, son aspectos repetitivos en los relatos de los/as docentes. El argumento de una profesora del PFP de la especialidad de Peluquería apunta en esta dirección: 
Mis evaluaciones son continuas, observación continúa del alumno. Yo hago mis exámenes teóricos y prácticos. Una evaluación continua, superviso trabajo, cuadernos, actitudes, puntualidad, la forma en que el alumno se exige a sí mismo, inquietud de aprender, eso me da mucho en que yo crea y confíe (Profesora, PFP Peluquería).

En el mismo sentido, incluimos la cita de una docente de PFP del perfil de Operaciones Auxiliares de Revestimiento en Construcción, en el que se refleja la importancia de la conducta y valores como aspectos valorados para la evaluación del alumnado:

[...] parto de los resultados de la evaluación inicial que les hago cuando vienen [...] tomo notas de su actitud y su progreso de cara a la evaluación. Yo valoro el esfuerzo, más que lo que aprendan, además se lo hago saber, para que sean más participativos [...] esto es un $40 \%$ de la nota, el otro $60 \%$ corresponde a los contenidos [...] bueno hay que tener en cuenta que las asignaturas van de dos en dos, entonces de ambas se saca una media, si una tiene un 4 y otra un 6, está aprobado (Tutora, PFP Construcción).

Finalmente, las afirmaciones del alumnado rescatadas en los grupos de discusión realizados en este trabajo, ratifican los aspectos valorados por los/as docentes descritos por ellos/as mismos/as. Así lo expresan diferentes alumnos que cursan el PFP del perfil de Construcción:

Por el temario que hemos visto (alumno 4); Y a partir del 4 te hacen media. Si tienes un 4 y 6 se te queda en un 5; Si ven que tienes interés te ayudan un puñado, pero pasan de ti como la mierda si ven que no tienes interés. (alumno 1); El comportamiento (alumno 3)

\section{Discusión y Conclusiones}

La finalidad con la que se diseñan ambos programas presenta matices diferentes en cada uno de ellos, a pesar de responder a una pretensión final común referida al reenganche socioeducativo y/o inserción laboral del alumnado en riesgo de exclusión. Así, mientras el PAI pretende reducir la situación de absentismo y abandono escolar temprano para favorecer la promoción del alumnado en el sistema educativo, el PFP pretende que el alumnado que lo cursa pueda desarrollar un desempeño profesional, posibilitándole su integración tanto social como laboral. Por tanto, este último tiene una finalidad más profesional que no se presenta en el PAI y que se plasma en la existencia de un Módulo de Formación en Centros de Trabajo en su diseño curricular, que tampoco lo incluye el PAI. En torno a la diferencia descrita entre ambos programas giran algunos de los hallazgos que muestran las prácticas evaluativas de los aprendizajes que permiten el reenganche del alumnado implicado a través de estos programas.

Desde el diseño curricular en ambos programas, recogido en la normativa al respecto, se indica que la evaluación será continua, formativa e integradora teniendo en cuenta los criterios de evaluación, los objetivos, las competencias y los resultados de aprendizaje, y esta descripción de la evaluación ha sido evidenciada en el desarrollo de los programas a través de los datos empíricos recabados.

En definitiva, cabe concluir que las prácticas de evaluación desarrolladas en los programas estudiados que están obteniendo éxito para responder al reenganche y la inclusión del alumnado presentan unas peculiaridades/atributos que responden a una evaluación para el aprendizaje. A continuación, se destacan algunas de sus características fundamentales: 
- Cada estudiante es considerado como único y, por lo tanto, la evaluación tiene en cuenta a cada sujeto, sus particularidades y trayectoria escolar;

- Son numerosos los aspectos a los que el profesorado atiende para evaluar a los/as estudiantes y sus aprendizajes. Entre ellos, caben destacar: a) destrezas y habilidades que resaltan la importancia de un aprendizaje útil y de aplicación real y práctica; b) conocimientos más conceptuales relacionados con las materias curriculares; c) valores y conductas que evidencien la implicación del alumnado con su aprendizaje, conductas que facilitan los aprendizajes y las enseñanzas, tales como la puntualidad, buenas relaciones en el aula, esfuerzo individual, respeto, etc. Por tanto, se trata de una evaluación que combina habilidades y competencias socio-emocionales (SÁNCHEZ-CALLEJA; GARCÍA-JIMÉNEZ; RODRÍGUEZ-GÓMEZ, 2016) con aspectos curriculares que se consideran básicos en el desarrollo integral del alumnado;

- Se hace uso de diferentes instrumentos de evaluación, como pruebas orales y escritas, tareas y actividades de clase, observación diaria, etc. Cada uno de estos instrumentos evalúa diferentes conocimientos, habilidades y actitudes, otorgándose a su vez una ponderación a cada uno de los instrumentos. Todas las evaluaciones realizadas ofrecen información global de los progresos y aprendizajes del alumnado. Los datos evidencian la importancia del uso de diferentes instrumentos para la evaluación del alumnado. Todos ellos, sin grandes distinciones, posibilitan y contribuyen a la evaluación global de los aprendizajes de los estudiantes que se pone en práctica en estos programas. Si bien, los resultados ponen de manifiesto que ante la variedad de instrumentos de evaluación que se utilizan para comprobar los aprendizajes del alumnado en los programas analizados, su adecuación responde a la finalidad que se persiga con ellos, así como del propio uso que se realice de los mismos (SANMARTÍ PUIG, 2020; CASTEJÓN et al., 2019);

- Las prácticas evaluativas forman parte de un continuo (MATEO; VLACHOPOULOS, 2013), que informa del progreso del alumnado, lo que permite tomar decisiones y ajustar la intervención educativa al proceso de aprendizaje y a las características y necesidades de los alumnos;

- La aplicación de los aprendizajes por parte del alumnado y la realización de ejercicios a nivel individual son los aspectos más presentes en las respuestas de los/as estudiantes -referidas a aquellos aspectos que realizan en clase y aquello que sus docentes evalúan- para el caso del PFP. Sin embargo, tales cuestiones ocupan un lugar más secundario en el caso del PAI. Esto estaría indicando la presencia de prácticas curriculares más tradicionales en el PAI que en el PFP cuya finalidad apunta en mayor medida a la inserción laboral del alumnado;

- Se ofrece información al alumnado acerca de su aprendizaje. Aspectos que tienen que ver con la devolución de resultados de la evaluación por parte de los/as docentes, con la identificación de las posibles mejoras que el alumnado pueda lograr, así como con la corrección de los ejercicios y exámenes están muy presentes tanto en lo que el alumnado resalta como cuestiones que realizan en clase como aquello que dicen evaluar los/as docentes.

Tal y como se ha puesto de manifiesto a lo largo de este trabajo, la evaluación del aprendizaje del alumnado en el marco de este tipo de programas debe responder a la diversidad existente entre el alumnado y que en muchas ocasiones responde a trayectorias de "idas y venidas", de "dejar de creer" en sus posibilidades, de "etiquetas" arrastradas, etc.

En esta línea, los resultados arrojados en este estudio ratifican el desarrollo de una evaluación en el aula, que defiende una evaluación para el aprendizaje donde a través del uso de varios instrumentos 
de evaluación que tienen como referente criterios personales y curriculares, se contribuye a fortalecer los aprendizajes del alumnado y se les ayuda a recuperar la confianza en sus posibilidades de aprender. De esta manera, "al sentir que pueden aprender" se empoderan y confían en sí mismos, aspectos estos que les sirven como motor para "redirigir" sus trayectorias vitales hacia una mayor implicación tanto con sus procesos formativos como laborales.

Asimismo, las conclusiones de otras investigaciones a nivel internacional sobre el impacto que estos programas ejercen sobre el reenganche, son coincidentes con los resultados presentados en este artículo. Resaltan los logros que adquiere el alumnado tras su paso por dichos programas (TE REIELE et al., 2017; REIMER; PANGRAZIO, 2020; SAVELSBERG; PIGNATA; WECKERT, 2017) entre los que se pueden destacar: desarrollo personal, reestructuración de la autoestima, actitud más positiva hacia la formación y ganas de aprender, acatamiento de normas de comportamiento y disciplina de trabajo, satisfacción personal, mejora de la asistencia y de los resultados académicos, desarrollo de habilidades sociales, incremento de la autonomía y la disposición y, cambio sobre las expectativas vitales.

Sin embargo, cabe alertar sobre el hecho de que al diversificar los instrumentos y los criterios de evaluación no se incurra en una reducción de los aprendizajes fundamentales necesarios para posibilitar la continuidad formativa de estos estudiantes. Pues en muchas ocasiones, se advierte que el éxito de los mismos, es parcialmente debido a una reducción considerable de los contenidos curriculares. De esta manera, habrá que desarrollar un currículum rico y relevante y que en coherencia se desarrolle una evaluación auténtica y para la enseñanza que contribuya a garantizar el derecho a una educación de calidad que favorezca, y no limite o hipoteque, el desarrollo personal, académico y social de los jóvenes.

Por tanto, el análisis presentado en este trabajo sobre dos programas de reenganche educativo/ formativo y/o laboral de jóvenes, contribuye al ámbito de estudio de estas realidades socio-educativas aportando experiencias en cuanto a prácticas curriculares que demuestran tener un impacto positivo en la mejora de las vidas de estos estudiantes. Esto supone una contribución que, sin duda, puede aportar conocimiento a nivel europeo y hacia el contexto Latinoamericano, en general, y brasileño, en particular, para vehicular políticas educativas y sociales que incluyan medidas y programas teniendo en cuenta aquellos aspectos que a nivel curricular están dando resultados positivos en otros contextos con realidades problemáticas muy similares.

\section{Contribución de las Autoras}

Problematización y Conceptualización: González-Barea E; Rodríguez-Entrena MJ; BolarínMartínez MJ; Metodología: Rodríguez-Entrena MJ; Bolarín-Martínez MJ; González-Barea E; Análisis: Bolarín-Martínez MJ; González-Barea E; Rodríguez-Entrena MJ; Redacción: González-Barea E; BolarínMartínez MJ; Rodríguez-Entrena MJ.

\section{Notas}

1. Disponible en: https://ec.europa.eu/eurostat/statistics-explained/index.php?title=Early_leavers_from_ education_and_training\#Overview, Acceso en: 23 jun. 2020.

2. Disponible en: https://www.ine.es/jaxiT3/Datos.htm?t=14506\#!tabs-tabla, Acceso en: 15 ene. 2020.

3. Disponible en: https://www.educacionyfp.gob.es/dam/jcr:7bd02364-3fd2-405f-b0d6-4fe05debbd38/seie-2020. pdf. Acceso en: 2 enero 2020. 
4. Creado en 2005 a través del Decreto n. 5.478, de 24 de junio de 2005, y desarrollado al siguiente año en el Decreto n. 5.840 de 13 de julio de 2006.

5. Del equipo docente en esta entidad y para los dos perfiles formativos se repiten dos docentes, que imparten en ambos perfiles. Los otros dos docentes imparten en el módulo asociado a unidades de competencias y son especialistas para cada uno de los perfiles.

6. Programa incluido en el Decreto n. 220/2015, de 2 de septiembre de 2015, por el que se establece el currículo de la Educación Secundaria Obligatoria en la Comunidad Autónoma de la Región de Murcia, cap. VIII (Equidad en la Educación), art. 28. Las materias Educación Plástica, Visual y Audiovisual y Música, así como Religión o Valores éticos serán desarrolladas en el Grupo de Referencia.

\section{Referencias}

ADAME, M. T.; SALVÀ, F. Abandono escolar prematuro y transición a la vida activa en una economía turística: el caso de Baleares. Revista de Educación, Madrid, n. 351, p. 185-210, 2010.

BOLARÍN, M. J. (coord.). Reenganche socioeducativo: diferentes realidades. Educatio Siglo XXI, Murcia, v. 38, n. 2, p. 11-16., 2020.

BOLARÍN, M. J.; RODRÍGUEZ-ENTRENA, M. J. Un nuevo programa para fortalecer el reenganche escolar: el programa de aprendizaje integral. In: MURILLO, F.J. Avances en democracia y liderazgo distribuido en educación: Actas del II Congreso Internacional de Liderazgo y Mejora de la Educación. Madrid: RILME, 2018. p. 57-60.

BOLÍVAR, A. Los nuevos currículos de la LOMCE. Un primer análisis crítico. Escuela, Madrid, v. 4007, n. 68, p. 28, 2014.

CASTEJÓN, J. et al. Técnicas e instrumentos de evaluación. In: LÓPEZ PASTOR, V. M. Evaluación formativa y comparada en Educación Superior. Propuestas, técnicas, instrumentos y experiencias. Madrid: Narcea, 2019. p. 65-92.

CEBREIRO, B.; FERNÁNDEZ MORANTE, M. C. Estudio de casos. In: SALVADOR MATA, F.; RODRÍGUEZ DIÉGUEZ, J. L.; BOLÍVAR BOTIA, A. Diccionario enciclopédico de didáctica. Málaga: Aljibe, 2004.

COMUNIDAD AUTÓNOMA DE LA REGIÓN DE MURCIA. Orden de 3 de septiembre de 2015. Normativa por la que la Consejería de Educación y Universidades regula los Programas Formativos Profesionales. Murcia: CARM, 2015a.

COMUNIDAD AUTÓNOMA DE LA REGIÓN DE MURCIA. Decreto n. 220, de 2 de septiembre de 2015. Reglamento que establece el currículo de la Educación Secundaria Obligatoria. Murcia: CARM, 2015b.

ESCUDERO, J. M. Fracaso Escolar, exclusión educativa. ¿De qué se excluya y cómo? Profesorado - Revista de Curriculum y Formación del Profesorado, Granada, v. 1, n. 1, p. 1-24, 2005.

ESCUDERO, J. M.; GONZÁLEZ, M. T. Estudiantes en riesgo, centros de riesgo: Dimensiones, interpretaciones e implicaciones prácticas. In: ESCUDERO MUÑOZ, J. M. Estudiantes en riesgo y centros escolares de riesgo: Respuestas educativas al alumnado en situaciones de vulnerabilidad. Murcia: Diego Marín, 2013. p. 14-46. 
FRADE, L. La evaluación por Competencias. México: Perseo, 2008.

GONZÁLEZ BAREA, E. M. Fracaso escolar, desenganche y aprendizaje-servicio. In: F. J. MURILLO (coord.). Avances en democracia y liderazgo distribuido en educación: Actas del II Congreso Internacional de Liderazgo y Mejora de la Educación. Madrid: RILME, 2018. p. 488-491.

HATTIE J. Visible learning: A synthesis of over 800 meta-analyses related to achievement. London: Routledge, 2009.

INGLÉS, C. J. et al. Conducta prosocial y motivación académica en estudiantes españoles de Educación Secundaria Obligatoria. Universitas Psychologica, Bogotá, v. 10, n. 2, p. 451-465, 2011.

LÓPEZ GONZÁLEZ, W. El estudio de casos: una vertiente para a investigación educativa. Educere, Mérida, v. 17, n. 56, p. 139-144, 2013.

MARCELO, M.; RIVERA, P. El docente universitario y su rol en la planificación de la sesión de enseñanza aprendizaje. En Blanco \& Negro, Lima, v. 8, n. 1, 2017 Disponible en: http://revistas.pucp.edu.pe/index. php/enblancoynegro/article/view/18958/19172. Acceso en: 30 nov. 2019

MARTÍNEZ-CARAZO, P. C. El método de estudio de caso: estrategia metodológica de la investigación científica. Pensamiento \& Gestión, Barranquilla, v. 20, p. 165-193, 2006.

MARTÍNEZ-SALGADO, C. El muestreo en investigación cualitativa. Principios básicos y algunas controversias. Ciência \& Saúde Coletiva, Rio de Janeiro, v. 17, n. 3, p. 613-619, 2012. https://doi.org/10.1590/ S1413-81232012000300006

MATEO, J.; VLACHOPOULOS, D. Reflexiones en torno al aprendizaje y a la evaluación en la universidad en el contexto de un nuevo paradigma para la Educación Superior. Educación XX1, Madrid, v. 16, n. 2, p. 183-208, 2013. https://doi.org/10.5944/educxx1.2.16.10338

MILES, M. B.; HUBERMAN, A. M.; SALDAÑA, J. Qualitative Data Analysis. A Methods Sourcebook. 3. ed. Thousand Oaks: Sage, 2014.

MINISTÉRIO DA EDUCAÇÃO. Programa nacional de integração da educação profissional com a educação básica na modalidade de educação de jovens e adultos. Brasília, DF: Secretaria de Educação Profissional e Tecnológica, ago. 2007. Disponible en: http://portal.mec.gov.br/setec/arquivos/pdf2/proeja_ medio.pdf. Acceso en: 13 oct. 2019

MINISTERIO DE EDUCACIÓN Y FORMACIÓN PROFESIONAL. Panorama de la educación. Indicadores de la OCDE 2019. Madrid: Instituto Nacional de Evaluación Educativa, 2019. Disponible en: https://www. educacionyfp.gob.es/dam/jcr:b8f3deec-3fda-4622-befb-386a4681b299/panorama\%20de\%20la\%20 educaci\%C3\%B3n\%202019.pdf. Acceso en: 13 ene. 2020

MONTANERO, M. Didáctica General. Planificación y práctica de la enseñanza primaria. Cáceres: Universidad de Extremadura/Servicio de Publicaciones, 2019.

MORENO-OLIVOS, T. Lo bueno, lo malo y lo feo: las muchas caras de la evaluación. Revista iberoamericana de Educación Superior, Ciudad de México, v. 1, n. 20, p. 84-97, 2010.

MORENO-OLIVOS, T. Evaluación del aprendizaje y para el aprendizaje. Reinventar la evaluación en el aula. Ciudad de México: Casa abierta al tiempo/Universidad Autónoma Metropolitana, 2016. 
NICOL, D.; THOMSON A.; BRESLIN, C. Rethinking. Fee back practices in higher education: a peer review perspective. Assessment \& Evaluation in Higher Education, Abingdon, v. 39, n. 1, p. 102-22, 2014. https:// doi.org/10.1080/02602938.2013.795518

NOGUEIRA, J.; PINEDA, C.; PINHEIRO, H. El Plan Nacional de Educación Brasileña (2014-2024) y los desafíos para el desarrollo local. Research, Society and Development, Itajubá, v. 7, n. 4, p. 1-21, e274169, 2018. https://doi.org/10.17648/rsd-v7i4.225

OCDE [ORGANIZACIÓN PARA LA COOPERACIÓN Y EL DESARROLLO ECONÓMICO]. La definición y selección de competencias clave. Resumen ejecutivo. [S. 1.]: OCDE, 2005. Disponible en: http://deseco.ch/bfs/deseco/en/index/03/02.parsys.78532.downloadList.94248. DownloadFile.tmp/2005. dscexecutivesummary.sp.pdf. Acceso en: 2 jul. 2005.

REIMER, K.; PANGRAZIO, L. Educating on the margins: young people's insights into effective alternative education. International Journal of Inclusive Education, London, v. 24, n. 5, p. 479-495, 2020. https://doi. org/10.1080/13603116.2018.1467977

SÁNCHEZ-CALLEJA, L.; GARCÍA-JIMÉNEZ, E.; RODRÍGUEZ-GÓMEZ, G. Evaluación del diseño del programa AedEM de Educación Emocional para Educación Secundaria. Revista Electrónica de Investigación y Evaluación Educativa, Valencia, v. 22, n. 2, 2019. https://doi.org/10.7203/relieve.22.2.9422

SANMARTÍ PUIG, N. Evaluar y aprender: un proceso único. Barcelona: Octaedro, 2020.

SANTOS GUERRA, M. A. La evaluación como aprendizaje. Cuando la flecha impacta en la diana. Madrid: Narcea, 2015.

SAVELSBERG, H.; PIGNATA, S. Y.; WECKERT, P. Second chance education: barriers, supports and engagement strategies. Australian Journal of Adult Learning, Canberra, v. 57, n. 1, p. 41-56, 2017.

TE RIELE, K. K. et al. Outcomes from flexible learning options for disenfranchised youth: what counts? International Journal of Inclusive Education, London, v. 21, n. 2, p. 117-130, 2017. https://doi.org/10.10 $80 / 13603116.2016 .1168878$

TRILLO, F. Competencias docentes y evaluación auténtica: ¿falla el protagonista? Perspectiva Educacional, Formación de Profesores, Valparaíso, v. 45, p. 86-102, 2005.

VILLEGAS, M. M.; GONZÁLEZ, F. La investigación cualitativa de la vida cotidiana. Medio para la construcción de conocimiento sobre lo social a partir de lo individual. Psicoperspectivas, Valparaíso, v. 10, n. 2, p. 35-59, 2011. Disponible en: http://www.psicoperspectivas.cl/index.php/psicoperspectivas/article/ viewFile/147/153\&a=bi\&pagenumber=1\&w=100. Acceso en: 24 feb. 2011.

\section{Sobre las Autoras}

Eva Maria González Barea es Licenciada en Pedagogía (1998) y Doctora en Antropología Social y Cultural (2003) por la Universidad de Granada. Profesora Titular en el Departamento de Didáctica y Organización Escolar, Universidad de Murcia. Miembro del Grupo de Investigación Equidad e Inclusión en Educación. Las líneas de investigación que desarrolla se enmarcan en temáticas sobre Fracaso Escolar, Abandono Prematuro, Exclusión Socio-educativa, Curriculum, Interculturalidad, Género y Diversidad. 
María Jesús Rodríguez Entrena es Licenciada en Pedagogía y en Psicopedagogía por la Universidad de Granada (2003). Doctora en Educación (2012) por la Universidad de Murcia. Profesora Titular en el Departamento de Didáctica y Organización Escolar, Universidad de Murcia. Miembro del Grupo de Investigación Equidad e Inclusión en Educación. Las líneas de investigación que trabaja son Exclusión Social y Educativa, Liderazgo Escolar, Formación del Profesorado, Alumnado Vulnerable e Innovación Pedagógica.

M. a José Bolarín Martínez es Licenciada en Ciencias de la Educación (1990) y Doctora en Pedagogía (1999) por la Universidad de Murcia. Especialista en Educación Infantil por la UNED (2000). Profesora Contratada Doctor en el Departamento de Didáctica y Organización Escolar de la Universidad de Murcia. Miembro del Grupo de Investigación Equidad e Inclusión en Educación. Las áreas de investigación desarrollada se inscriben en los ámbitos de Fracaso Escolar, Abandono Prematuro, Exclusión Socio-educativa, Coordinación Docente y Ambientes de Aprendizaje en Educación Infantil.

Recibido: 25 Mayo 2020

Aceptado: 25 Nov. 2020 